\title{
Spectral Width Study in a Wavelength Detector
}

\section{Estudio de la anchura espectral en un detector de longitud de onda}

\author{
A.M. Alaíz-Gudín ${ }^{1 *}$, A.P. González-Marcos ${ }^{1}$ \\ 1. ETSIT, Universidad Politécnica de Madrid, Avda Complutense, 30, 28040 Madrid \\ $\left.{ }^{*}\right)$ E-mail: am.alaiz@alumnos.upm.es
}

Received: 19/05/2015 Aceptado: 18/04/2016

DOI: $10.7149 /$ OPA.49.2.48540

\begin{abstract}
:
In this paper, we describe different use configurations of a wavelength detector and the main parameters of an optical bistable sensor. Mainly we analyze by simulation tools the influence of the spectral width of each signal involved in our wavelength detector, namely the LDD -Laser Diode Detector-. In particular, we study the spectral width effect of the signal to be detected. The LDD, based on the bistable behavior of semiconductor lasers, has an internal continuous wave laser that allows the calibration of the sensor. Also, the effect of the line width is analyzed for this CW laser. It is shown that, for the different types of signals, the detector achieves accuracy near to $0.001 \mathrm{~nm}$, working with typical manufacture parameters values of the laser diodes and with wavelengths centered in the optical communications C-band.
\end{abstract}

Key words: Linewidth, Wavelength Detector, Laser Diode, Bistability, Optical Sensor

\section{RESUMEN:}

Se describen las diferentes configuraciones de uso de un detector de longitud de onda y los principales parámetros de un sensor óptico biestable. Se analiza en el dominio óptico mediante herramientas de simulación, la influencia del ancho espectral de las diferentes señales que intervienen en el detector de longitud de onda propuesto por los autores. El detector de longitud de onda denominado LDD (del inglés Laser Diode Detector) está basado en el comportamiento biestable de los láseres de semiconductor. Se estudia el efecto de la anchura espectral de la señal a detectar, y la del láser de onda continua que forma parte del dispositivo LDD. Se demuestra, en el estudio de los diferentes tipos de señales presentes, que se mantiene una precisión del detector cercana a $0.001 \mathrm{~nm}$, con valores típicos de fabricación de los diodos láser y a longitudes de onda centradas en la banca $\mathrm{C}$ de comunicaciones ópticas.

Palabras clave: Ancho de Línea, Detector de Longitud de Onda, Diodo Láser, Biestabilidad, Sensor Óptico

\section{REFERENCIAS Y ENLACES}

[1] Wei Xia, Xuzong Chen, "Recent developments in fiber-based optical frequency comb and its applications”, Meas. Sci. Technol., 27, No.4 (2016) http://dx.doi.org/10.1088/0957-0233/27/4/041001

[2] Elisa Garmire, "Resonant Optical Nonlinearities in Semiconductors". IEEE J. Sel. Top. Quantum Electron, 6, 1094-110 (2000) http://dx.doi.org/10.1109/2944.902158

[3] H. Kawaguchi, "Absorptive and dispertive bistability in a Fabry-Perot laser diode amplifier", Opt. Quantum Electron., vol. 19, pp. s1-s36(1987) http://dx.doi.org/10.1007/BF02034349

[4] Hitoshi Kawaguchi. "Bistable Laser Diodes and Their Applications: State of the Art." IEEE J. Sel. Topics Quantum Electron. 3, 1254-1270 (1997) http://dx.doi.org/10.1109/2944.658606 
[5] A. Takada, T. Sugie, M. Saruwatari, "High-Speed Picosend Optical Pulse-Compression from GainSwitched 1.3-mu-m Distributed Feedback-Laser Diode (DFB-LD) Through Highly Dispersive SingleMode Fiber", J. Lightwave Technol. 5, , 1525-1533 (1987) http://dx.doi.org/10.1109/JLT.1987.1075418

[6] W. Qian, "High-sensitivity temperature sensor based on an alcohol-filled photonic crystal fiber loop mirror". Opt. Lett. 36, 1548-1550 (2011) http://dx.doi.org/10.1364/OL.36.001548

[7] J.A. Martín Pereda. “Sistemas y Redes Ópticas de Comunicaciones. Pearson España, 229-236 (2004)

[8] A. M. Alaíz, A.P. González. "Wavelength-Shift Detection by Semiconductors Laser", II Congreso DESEi+d, 121-128, (2014)

[9] A. Yariv, P. Yeh. Photonics: Optical Electronics in Modern Communications. 6th ed., Oxford University Press, (2007)

[10] C.H. Henry, "Theory of the Linewidth of Semiconductor Lasers IEEE J. Quantum Electron, QE-18, 259264. (1982)

[11] Antonio M. Alaíz, Ana P. González. “Optical M.I.D.O. Device”, IONS Valencia 2015.

[12] D. A. B. Miller, D. S. Chemla, T. C. Damen, T. H. Wood, C. A. Burrus, A. C. Gossard and W. Wiegmann, "The quantum well self-electrooptic effect device: Optoelectronic bistability and oscillation, and self linearized modulation", IEEE J. Quantum Electron., vol. QE-21, 1462$1476,(1985)$ http://dx.doi.org/10.1109/JQE.1985.1072821

[13] A. Hurtado, A. González-Marcos, J.A. Martín-Pereda. "All-optical logic gates with 1550nm Fabry-Perot and distributed feedback semiconductor laser amplifiers". IEEE Spanish Conference on Electron Devices. (2005) http://dx.doi.org/10.1109/SCED.2005.1504388

[14] A. Hurtado, A. González-Marcos, José A. Martín-Pereda. "Wavelength monitoring with semiconductor laser amplifiers. Second European Workshop on Optical Fibre Sensors". (2004)

[15] Zhiyong Dai, Bo Wu, Zengshou Peng, Zhonghua Ou, Yongzhi Liu. "Stable Narrow Linewidth Ring Fiber Laser with a Passive Fiber Bragg Grating Fabry-Perot Etalon and a Fiber Saturable Absorber". International Conference on Optical Instruments and Technology: Microelectronic and Optoelectronic Devices and Integration. Proc. SPIE 7158, 715817 (2009) http://dx.doi.org/10.1117/12.806925

[16] "Fujitsu, University of Tokyo Develop World's First 10Gbps Quantum Dot Laser Featuring Breakthrough Temperature-Independent Output", http://www.fujitsu.com/global/about/resources/news/press-releases/2004/0910-01.html

[17] Marek Osinski, "Linewidth Broadening Factor in Semiconductor Lasers" - An Overview. IEEE Journal of Quantum Electronics, Vols. QE-23, 9-29. (1987)

\section{Introducción}

En los láseres monofrecuencia es importante que la anchura de línea sea lo más pequeña posible. Se ha de alcanzar con un dispositivo en el que se busca la mayor sencillez y un bajo coste de componentes de fabricación que facilite su desarrollo para la enorme cantidad de aplicaciones que tiene, como son las comunicaciones, los sensores o los relojes ópticos [1]. En este caso, el objetivo es encontrar un sensor óptico con gran sensibilidad y que al mismo tiempo mantenga baja la complejidad y el coste, lo que es posible con los diodos láser. El sensor permite monitorizar la longitud de onda de la señal que recibe en relación a otra de referencia, la variación respecto a la de referencia se traduce en un cambio de respuesta del dispositivo. Se produce así un cambio de nivel de la potencia óptica a la salida del dispositivo. Esta variación en la longitud de onda puede estar provocada por la diferencia de la magnitud que queremos medir o por la presencia de la sustancia sobre la que queremos intervenir.

Gracias a la no-linealidad de los dispositivos biestables, Bistable Device (BD), que utilizamos, podemos obtener gracias a una mínima potencia óptica un sensor con gran precisión y bajo consumo. Los BDs empleados en la simulación presentada en este trabajo son láseres Fabry Perot (FP) con estructuras Multiple Quantum Well (MQW) en su región activa. Los materiales semiconductores presentan una elevada 
no linealidad óptica resonante [2], que permite el diseño de una gran variedad de dispositivos fotónicos basados en ellos. Los láseres de semiconductor (LD), en sus diversas configuraciones de la cavidad resonante -FP [3], Vertical Cavity Surface Emitted Laser (VCSEL)[4], Distributed FeedBack (DFB) [5] y anillos [6]- y de medio activo -bulk, Quantum Well (QW), Multiple Quantum well (MQW) y Quantum Dot (QD) [7]-, es uno de los dispositivos optoelectrónicos con mayor número de aplicaciones gracias a su pequeño tamaño, a su capacidad de emitir en longitudes de onda dentro de los márgenes más empleados en diferentes campos de aplicación, así como su facilidad de fabricación y comercialización.

La descripción de la biestabilidad, en una configuración resonante de FP y de su aplicación a la estructura que conforma el detector LDD (Laser Diode Detector), ha sido realizada en la publicación [8]. Interesa que el LDD sea robusto en cuanto a las variaciones de la anchura espectral de la señal a monitorizar y de los elementos que lo constituye. Esta robustez es en la que se centra este estudio.

\section{Configuración del detector de longitud de onda}

La señal a la entrada del LDD es de tipo óptico, al igual que su respuesta, y puede proceder de la fuente de referencia del LDD o de una fuente externa. La señal se introduce y extrae del medio bajo estudio mediante una fibra. La fibra puede actuar como sensor o captar la reflexión y transmisión de la señal que incide directamente sobre dicho medio (figura 1). Al calibrar el dispositivo se asocia el nivel de salida a la presencia o no de una determinada longitud de onda, y así podemos, gracias a la sensibilidad que presenta el LDD, detectar inmediatamente que el medio que estamos estudiando induce variaciones en la longitud de onda.

La idea del funcionamiento de este tipo de sensor es la siguiente: por medio de un ajuste del mismo y un correcto calibrado, vamos a poder tener el dispositivo centrado en una determinada longitud de onda. Al ser capaces de percibir pequeñas variaciones de esa longitud de onda, podemos detectar cualquier cambio en el medio en el que trabaje otro sensor, por ejemplo de fibra, de manera inmediata (ver configuración de la figura 1a). También es válido obtener la señal procedente de otra fuente externa al dispositivo, directamente del medio ambiente o de una línea de comunicaciones o red de sensores, y comprobar la existencia de una determinada longitud de onda, que se correspondería con la de calibración del LDD, tal como se muestra en la figura $1 \mathrm{~b}$.
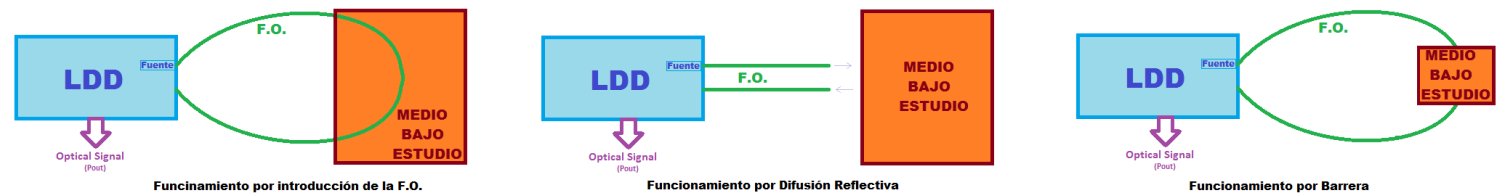

a) Fuente en el LDD

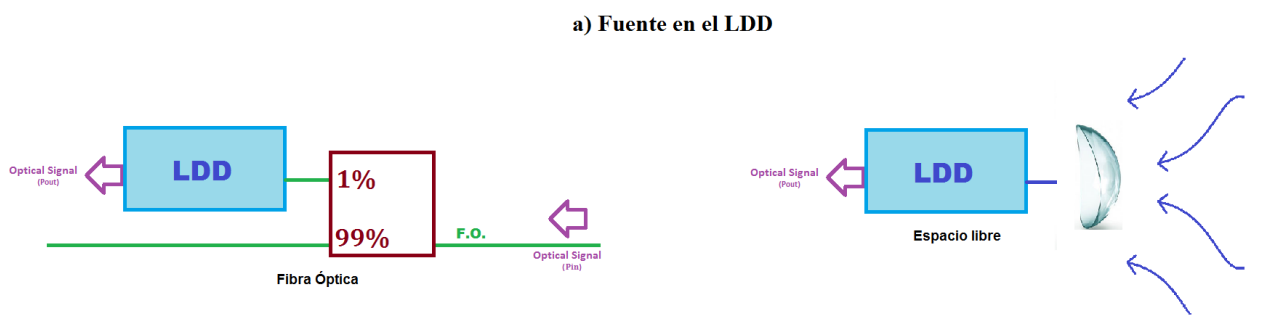

b) Fuente externa

Figura 1 Tipos de entrada al LDD: a) con la fuente en el LDD; b) con una fuente externa

Si al concepto del LDD con una sola longitud de onda le damos la capacidad de realizar un barrido dentro de un margen de longitudes de onda, tendríamos un detector sintonizable.

Sin embargo, hay escenarios en los que no nos interesa la sintonía del detector, sino que el detector actúe como sistema de alarma. En estos casos, se fija la longitud de onda para un valor determinado y cualquier variación se vería reflejada. Dichas variaciones se producen a consecuencia de cambios en parámetros habituales, por temperatura o presión, o incluso por modificaciones del medio, de manera que 
determinados elementos que anteriormente no estaban en el ambiente se pueden ver después. Elementos que se quiere identificar como por ejemplo algunos gases perjudiciales.

Como sistema de alarma podemos trabajar en dos configuraciones, o bien nos encontramos en un medio hostil o trabajando en un entorno donde el margen de funcionamiento correcto es muy pequeño, de forma que mientras detectemos la longitud de onda deseada estamos seguros, figura 2(a). En el otro ejemplo, la detección de determinada longitud de onda nos avisa de una situación de peligro. Podemos contar con un LDD a modo de analizador para que nos indique la presencia de elementos peligrosos, útil para detectar tóxicos o materiales nocivos (figura 2(b)).
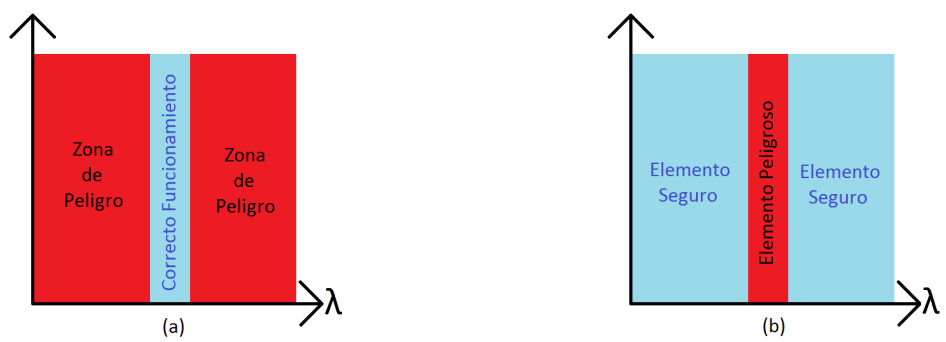

Figura 2 Tipos de Funcionamiento del Sensor como Sistema de Alarma

\subsection{Margen de sintonía y precisión}

El funcionamiento de un detector de longitud de onda se caracteriza por el rango de longitudes de onda que es capaz de detectar y la precisión con que lo hace, y en un segundo plano tendríamos la capacidad de sintonía del detector. En el caso que nos ocupa, el sensor nos indica la presencia de una determinada longitud de onda que la fijamos con la fuente LD interna del detector. Con una fuente LD sintonizable el detector tiene capacidad de sintonía.

Si consideramos que el sensor de longitud de onda está basado en la respuesta de una cavidad FP, una primera aproximación al límite del margen de sintonía del sensor nos viene dado por [9]

$$
\Delta v_{(1 / 2)}=\Delta v / F
$$

donde $\mathrm{F}$ es la fineza de un etalón de Fabry-Perot, parámetro que se emplea como medida de la resolución de los analizadores de espectros ópticos basados en interferómetros de FP; y $\Delta v$ se corresponde con la separación entre modos de la cavidad.

Considerando los valores típicos de un diodo láser de FP donde las reflectividades no son mayores de 0,3 e iguales en ambas caras, obtenemos

$$
\Delta v_{(1 / 2)}=0.4 \Delta v
$$

con una la longitud de la cavidad de unos $0,5 \mathrm{~cm}$, y un valor del índice de refracción lineal típico del material semiconductor para láseres de tercera ventana: $n=3$, tenemos un margen de sintonía

$$
\Delta v_{(1 / 2)}=4 \mathrm{GHz}
$$

En el caso que se plantea en este trabajo, el analizador de espectros equivalente se sintoniza con la propia señal que se quiere identificar. El material semiconductor de los diodos láser tiene un índice de refracción no-lineal especialmente significativo cuando se trabaja en un régimen de corriente de polarización cercano a la corriente umbral del dispositivo. De $t$ forma que es necesario realizar un estudio más detallado de cómo se modifica el parámetro $\Delta v$ de la ecuación (2), que viene dado para una incidencia normal por

$$
\Delta v=\mathrm{c} / 2 \mathrm{nd}
$$

donde el índice de refracción -n- depende de la potencia inyectada en la cavidad de FP del diodo láser, permitiendo un comportamiento biestable en función de la potencia de entrada al BD; c es la velocidad de la luz y d la longitud de la cavidad resonante. 
El grado de precisión depende del ancho de línea de los dispositivos biestables. La zona activa LD-FP está fabricada con múltiples capas cuánticas -MQW- cuyo ancho de línea se corresponde con el ancho de cada uno de los modos de la cavidad FP y que, de acuerdo con Henry [10], depende también de la potencia que se aplique y de la corriente de polarización de los diodos láser que configuran los dispositivos biestables. Por lo que respecta al comportamiento del sensor, el ancho de línea afecta de manera directa tanto a las necesidades de funcionamiento del sensor como a su capacidad de detección.

\section{Función de Transferencia del LDD}

El sensor es una aplicación del dispositivo MIDO (Multiple Input Digital Output) que fue presentado en [11]. Su función de transferencia $\mathrm{P}_{\text {out }}\left(\mathrm{P}_{\text {in }}\right)$ es similar a la que presentaban los dispositivos Self Electrooptic Effect Device (SEED) en su comportamiento biestable [12]. En la aplicación que aquí mostramos, se fijan a priori los parámetros de corriente de polarización de los dispositivos biestables y la desintonización inicial de fase, y no se modifican posteriormente. El comportamiento de los dispositivos biestables también ha sido demostrado con LD-DFB (Distributed FeedBack) en [13]. En futuros trabajos se describirá el LDD con este tipo de estructuras y otras nuevas.

Brevemente, para comprobar la versatilidad del dispositivo MIDO, en la figura 3 podemos ver diferentes respuestas conseguidas por medio de la variación de la $\mathrm{I}_{\text {bias }}$ de uno de los BD (Bistable Device), que forman parte del mismo.

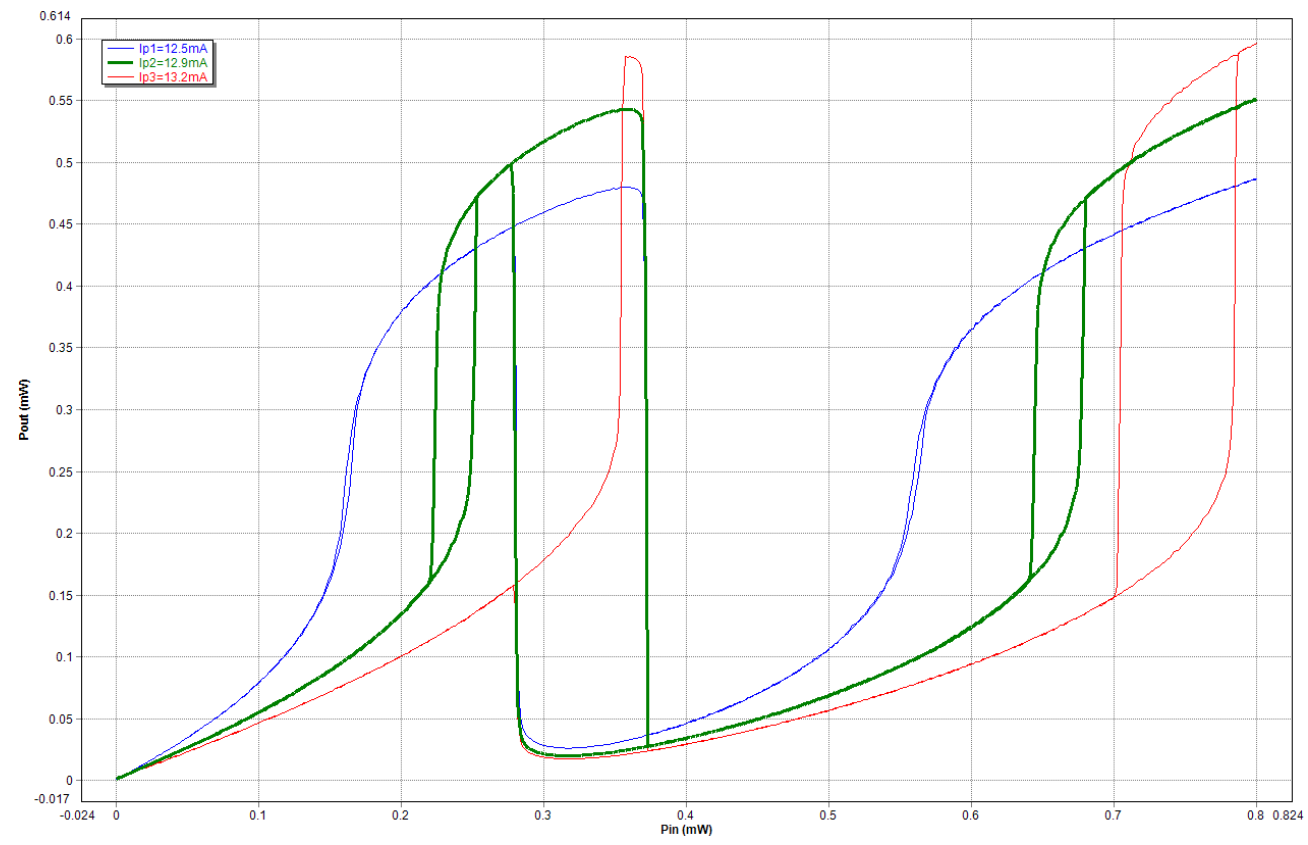

Figura 3 Diferentes Respuestas del Dispositivo MIDO

Como puede observarse en las tres funciones de transferencia representadas en la figura 3, en cada transición de una salida de bajo nivel $\left(\mathrm{P}_{\text {out-OFF }}\right)$ a una de alto nivel $\left(\mathrm{P}_{\text {out-oN }}\right)$ se forma un ciclo de histéresis. Dejando de lado los casos extremos en los que apenas se forman los ciclos de histéresis y en el que se produce el segundo ciclo antes de que finalice el primero, fijándonos en el caso de $\mathrm{I}_{\mathrm{p} 2}$ (representada en color verde), vemos que se forman ciclos de histéresis en el sentido contrario a las agujas del reloj en las

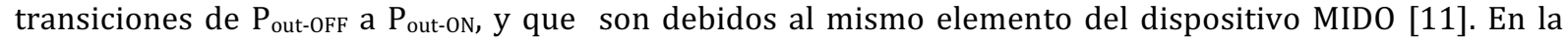
transición de $\mathrm{P}_{\text {out-on }}$ a $\mathrm{P}_{\text {out-off }}$ el ciclo de histéresis que aparece es en el sentido de las agujas del reloj. Sólo como ejemplo, para ver cómo varía la respuesta con corrientes de polarización mayores o menores a $\mathrm{I}_{\mathrm{p} 2}$, en la figura se muestran las funciones de transferencia para el caso de $\mathrm{I}_{\mathrm{p} 1}$, menor que $\mathrm{I}_{\mathrm{p} 2}$, y el caso de $\mathrm{I}_{\mathrm{p} 3}$, mayor que $\mathrm{I}_{\mathrm{p} 2}$. Para corrientes de polarización menores, los ciclos de histéresis se van cerrando, haciéndose apenas perceptibles los levógiros. En el caso de la más grande de las tres corrientes, la $\mathrm{I}_{\mathrm{p} 3}$, podemos comprobar cómo se puede llegar a que el ciclo de histéresis dextrógiros se produzca antes de que finalice el primer ciclo en el sentido contrario a las agujas del reloj, quedando una curva muy distinta 
de los dos casos anteriores. En cualquier caso, se observa que el punto de transición de $\mathrm{P}_{\text {out-on }}$ a $\mathrm{P}_{\text {out-ofF }}$ requiere en todos los casos la misma potencia de entrada $\mathrm{P}_{\text {in }}$ igual a $0.37 \mathrm{~mW}$.

Ahora, la función que define al detector de longitud de onda y que nos permite entender su funcionamiento es la potencia de salida en función de la frecuencia de las señal de entrada $\mathrm{P}_{\text {out }}(v)$. A continuación, para observar cómo afecta la variación de la longitud de onda al dispositivo MIDO, en la figura 4 se muestra el resultado de variar la longitud de onda de la señal de entrada, trabajando sobre la función de transferencia de la figura 3 correspondiente a la corriente $\mathrm{I}_{\mathrm{p} 2} \mathrm{y}$ en el punto donde la potencia de entrada $\mathrm{P}_{\text {in }}$ es de $0.5 \mathrm{~mW}$, más que necesaria para poder observar los efectos.

La figura 4 muestra una función con saltos abruptos producidos por la biestabilidad de los elementos que conforman el dispositivo MID0, muy similar a la demostrada en [14]. En ella vemos que podemos trabajar en dos configuraciones cuando se emplea el MIDO como detector de longitud de onda en su diseño como LDD: cuya interpretación de la respuesta del dispositivo es tal que dice que si el dispositivo pasa al estado de $\mathrm{P}_{\text {out-on }}(>0.45 \mathrm{~mW})$ se puede asegurar que en la señal de entrada al LDD tenemos una señal óptica de frecuencia $193.0877 \mathrm{THz}(1552.62 \mathrm{~nm})$ con un error de $\pm 3.58 \mathrm{GHz}(28.82 \mathrm{pm})$; y otra, en la que si el dispositivo se mantiene $\mathrm{P}_{\text {out-OFF }}(<0.15 \mathrm{~mW})$ tenemos una señal de entrada al LDD con una frecuencia de 193.0979THz $(1552.54 \mathrm{~nm})$ con un con un error de $\pm 6.3 \mathrm{GHz}(50.65 \mathrm{pm})$.

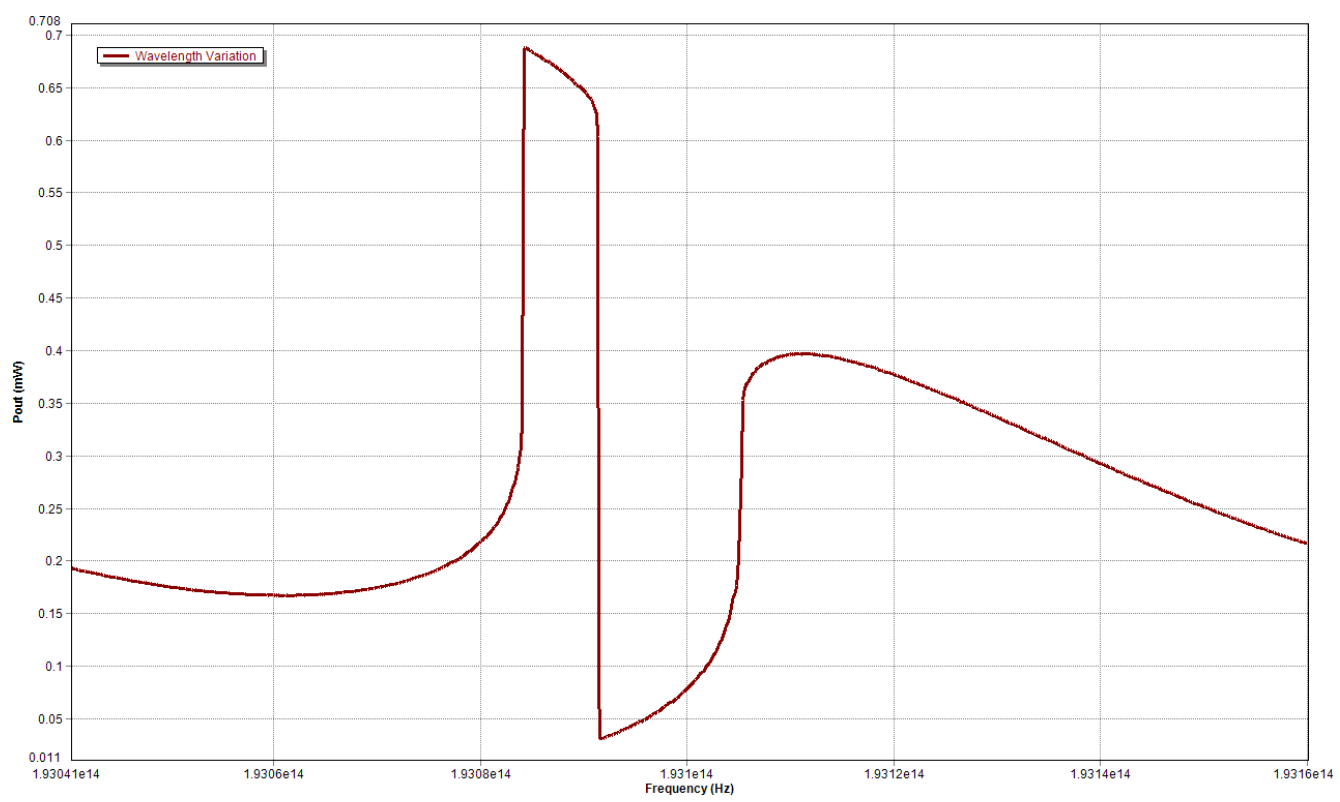

Figura 4 Respuesta del Dispositivo MIDO ante variación de la Longitud de Onda

Gracias a la configuración del dispositivo MIDO que utilizamos en este caso, podemos obtener el comportamiento que más adelante veremos.

\section{Materiales y métodos. Sección Experimental.}

Para llevar a cabo el estudio, hemos aprovechado las ventajas de las herramientas CAD -Computer Aided Design-, que permiten comprobar el comportamiento del diseño de sistemas antes de realizar las costosas pruebas experimentales, costosas no sólo en equipos sino en tiempo de búsqueda de las condiciones de funcionamiento. En este trabajo nos hemos servido de la herramienta CAD VPIphotonics ${ }^{\mathrm{TM}}$, que permite el diseño, implementación y simulación de diversas redes de fibra óptica y componentes fotónicos, por medio de una librería predefinida y actualizada de las nuevas tecnologías. Una de sus ventajas, necesarias para simular dichos efectos, es la capacidad de tener en cuenta el transcurso del tiempo para poder simular los efectos de la biestabilidad.

\subsection{LDD como sensor}

En la Figura 5 se muestra esquemáticamente el funcionamiento y la configuración del sensor empleado para el estudio publicado en este artículo. Como vimos en [8], y puede inferirse de la figura 3, a la salida 
del sensor tendremos un nivel alto de manera constante. Al tener a la entrada del sensor una señal con determinada longitud de onda, el nivel pasará a un nivel bajo de una potencia prácticamente nula. El sensor está formado por un láser de onda continua (CW Laser) -encargado de mantener dicho nivel de potencia constante a la salida- y dos dispositivos biestables que propiciarán el salto al nivel bajo cuando la señal de entrada tenga las características deseadas.

Cuando se utilizan detectores de uno o varios fotones sabemos que la energía de cada uno de ellos puede variar y, sin embargo, en la conversión a energía eléctrica perdemos la información de la longitud de onda de la señal. Si los fotones de la señal tienen como origen una fuente de fluorescencia o bioluminescencia, difícilmente la anchura espectral de la señal detectada será cero, pero podemos asegurar que la detección de una longitud de onda determinada indica la presencia de una sustancia. Igualmente, cuando las fuentes son artificiales como con los diodos láser convencionales, por su propio principio de funcionamiento nunca son monofrecuencia. Evidentemente, en este último caso la investigación y desarrollo actual está dando lugar a nuevas estructuras que permitan construir láseres con anchuras espectrales cada vez más pequeñas.

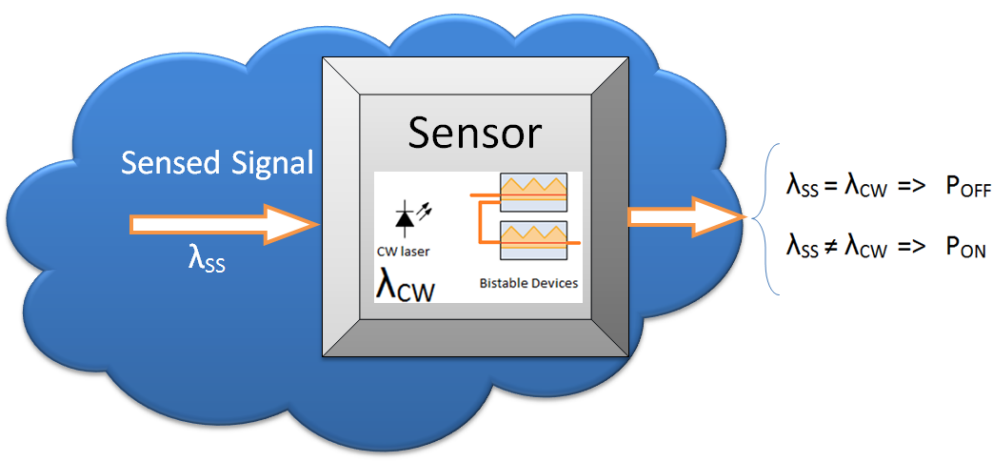

Figura 5 Esquema del Sensor

Con el sensor diseñado discriminamos la longitud de onda en el dominio óptico previamente a la detección o conversión de la señal óptica en señal eléctrica, lo que nos permite comprobar su presencia. De acuerdo con lo anterior, es interesante conocer cómo es el comportamiento del LDD con señales y anchos de línea diferentes, así como analizar si el detector es capaz de funcionar en un entorno poco amigable debido a la presencia de una señal de fondo óptica o señal de ruido.

\subsection{Estudio del ancho de línea}

En este apartado vamos a proceder a mostrar los resultados del estudio de la variación del ancho de línea en los distintos elementos que nos conciernen.

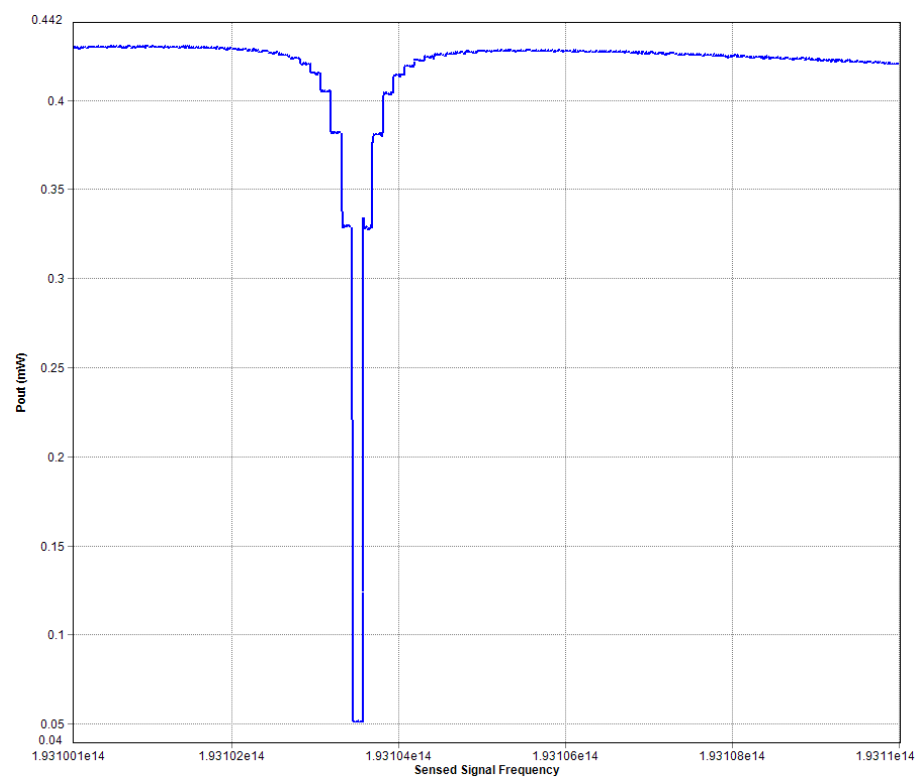

Figura 6 Frecuencia_SS - Pout_Sensor eje-x Frecuencia Señal Sensada $(\mathrm{Hz})$ 
En la Figura 6, podemos observar un caso ideal en el que tenemos un ancho de línea, tanto del CW láser $(0.4 \mathrm{~mW}, 193.1035 \mathrm{THz})$ que forma parte del sensor como de la Señal Sensada -SS- $(0,05 \mathrm{~mW})$ ideal, igual a cero. Lo que muestra la gráfica es una relación de la variación de la frecuencia de laSS respecto a la Potencia de Salida de nuestro Sensor. El eje x de frecuencia de la SS representado se corresponde con un barrido en el margen de longitudes de onda de 1552.52-1552.44nm. Vemos que cuando la señal es igual a las del láser de CW se produce un corte de señal, con $\mathrm{P}_{\text {out-ofF }}$

Pero esta situación ideal es difícil que se dé, por lo que a continuación, en la figura 7, se muestra la zona de interés, centrada en la longitud de onda a detectar, aumentada, esto es, en un margen de frecuencias más reducido con la misma cantidad de potencia óptica involucrada. Para poder realizar una buena comparativa de comportamiento se han representado tres casos simultáneamente:

a) El caso ideal anterior, con las anchuras de línea de las señales ópticas involucradas igual a cero

b) Un ancho de línea de $10 \mathrm{KHz}$ tanto para el CW Láser como para la anchura de la SS. En [15] podemos verificar que se han alcanzado anchos de línea menores de dicho valor para láseres similares de los que nos podríamos servir en el futuro para la realización del LDD experimental

c) Para un ancho de línea de $10 \mathrm{KHz}$ en el CW Láser y un ancho de línea de $125 \mathrm{MHz}$ de la SS.

En el segundo caso, solo se perciben mínimas oscilaciones a la salida del dispositivo que no varían su capacidad de detección.

En el tercer caso, podemos observar una gran cantidad de oscilaciones que pasan del nivel alto al bajo, pero seguimos teniendo la bajada de potencia que podríamos aprovechar para seguir manteniendo la capacidad de detectar y medir.

La precisión que obtenemos en este caso concreto podemos ver que sería de $1.06 \mathrm{pm}$.

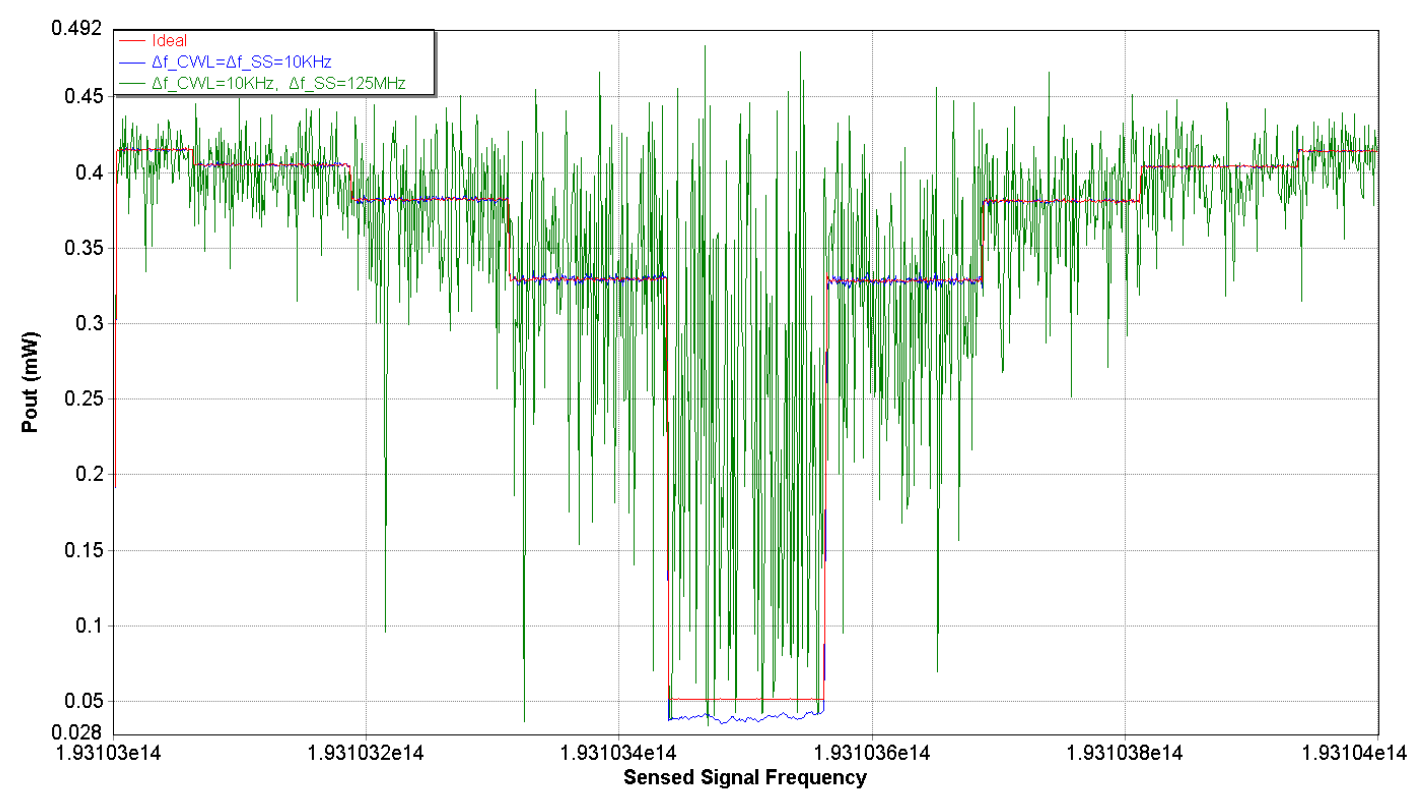

Figura 7 Zona de interés de la figura 6 con diferentes anchos de línea de los láseres

\section{2.c. Efectos del Ruido}

Pasamos a estudiar el efecto de tener un canal ruidoso. En cuanto al modelo de simulación, sería como añadir un Ruido Blanco Gaussiano a la entrada.

En la figura 8, en el gráfico pequeño, se muestra el espectro de la SS, generada por un láser sintonizable de un solo modo y la señal de ruido blanco generada. Ambas se suman en el dominio óptico e internamente se sumaran a la señal del láser de CW. En la figura se comprueba la diferencia en la señal de salida del LDD entre tener y no tener dicho ruido cuando se realiza el barrido de la señal a detectar. 


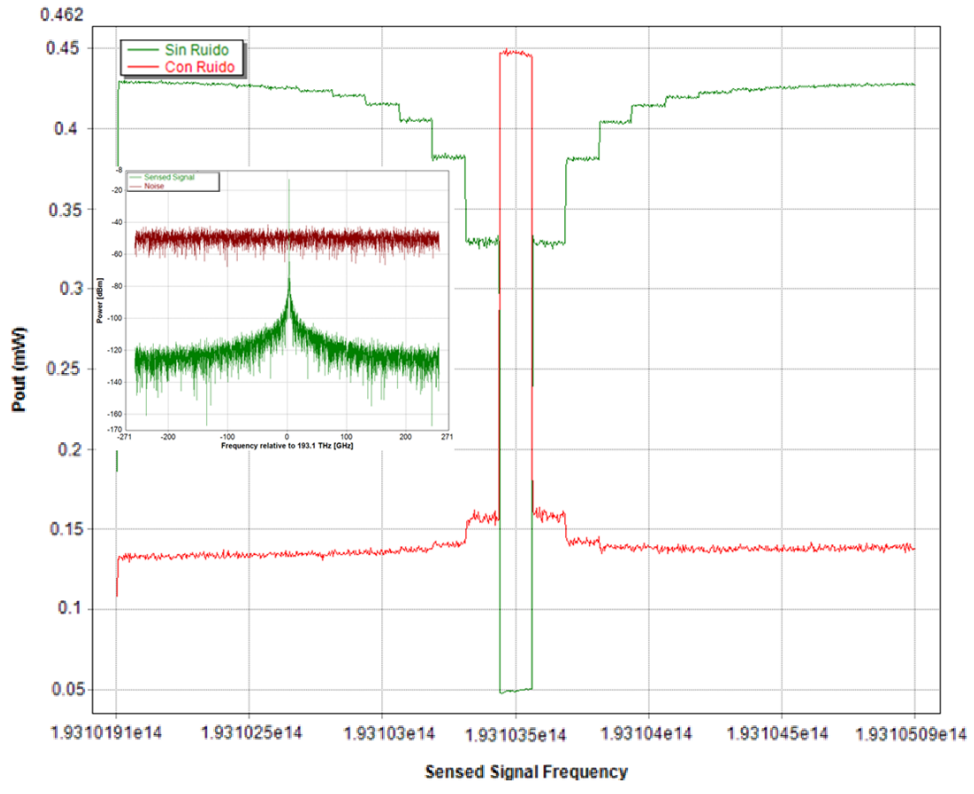

Figura 8. Comparación: respuesta con y sin ruido (se incluye, en la imagen pequeña, el espectro de la señal y del ruido a la entrada)

Podemos observar cómo cambia la respuesta $\mathrm{P}_{\text {out }}(v)$ del dispositivo, pero en ambos casos somos capaces de identificar la presencia de la longitud de onda a la que se tiene calibrado el dispositivo. Con la presencia de ruido de fondo no perdemos la capacidad de detección, ya que seguimos teniendo una variación de un estado bajo a otro alto al incidir una SS con la longitud de onda esperada por el sensor. El ruido sitúa al dispositivo en un punto de trabajo tal que mientras no hay señal de la deseada el LDD se encuentra en estado $\mathrm{P}_{\text {out-OFF }}(<0,15 \mathrm{~mW})$, pasando a $\mathrm{P}_{\text {out-ON }}(>0,4 \mathrm{~mW})$ al detectar la $\lambda$ deseada.

En la figura 9, mostramos el efecto que tiene un ruido mucho mayor y podemos comprobar que la robustez del dispositivo permite que se siga pudiendo detectar.

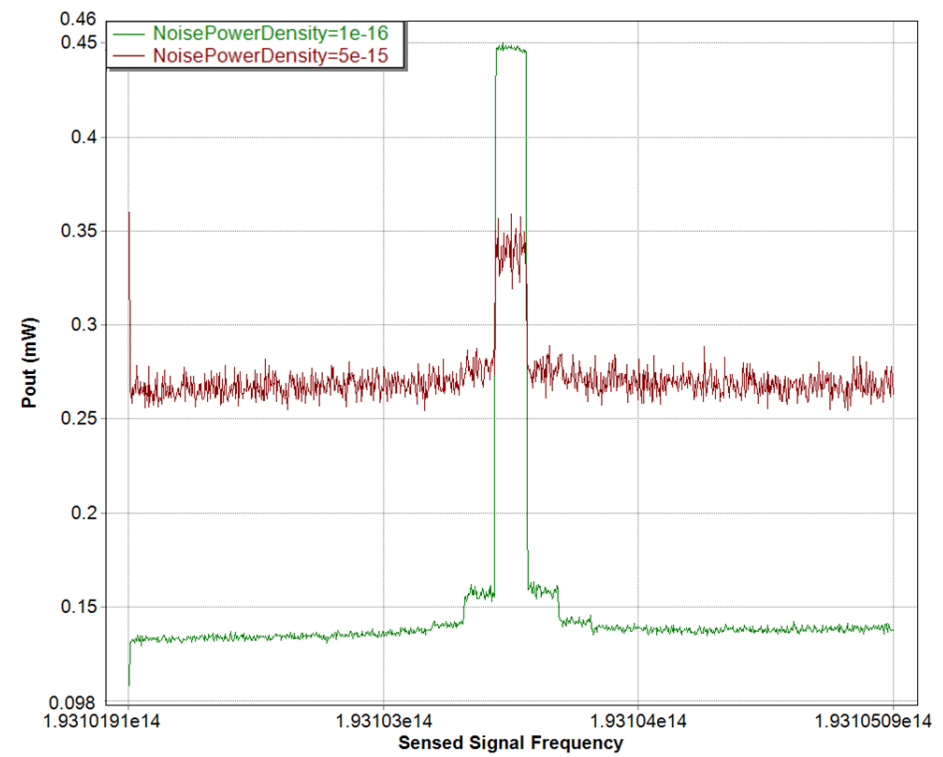

Figura 9 Comparación del Sensor con existencia de mucho ruido

\section{Resultados y Discusión}

Se ha demostrado cómo el sensor permite trabajar con la precisión definida en [8], de 0,001nm, independientemente del efecto que produce el ancho de línea de la Señal Detectada o del CW láser del detector de longitud de onda. Aún en el peor de los casos, en los que haya una señal con gran ancho de línea, el sensor sigue funcionando correctamente gracias al constante alcance de un nivel de potencia de salida prácticamente nulo a la longitud de onda de calibración. 
La presencia de ruido de fondo nos sitúa en la zona alta de la respuesta del detector, según la figura 4, y la explicación de esto queda fuera del alcance de este artículo. Sin embargo, sí podemos decir que hemos comprobado cómo permite poder darle otra configuración funcional al sensor, en el que el comportamiento sea al contrario del mostrado aquí: tendremos así un nivel prácticamente nulo salvo cuando detectemos una señal con determinada longitud de onda, y en ese caso se produciría un incremento de potencia a la salida del detector que nos situaría en el nivel alto.

Con respecto al problema de la temperatura, que afecta a la biestabilidad, gracias a las tecnologías actuales, como por ejemplo [16], y a la posibilidad del diseño de dispositivos en circuitos integrados, es más fácil disponer de un dispositivo cuya respuesta no dependa tanto de la temperatura, y además permitirá controlar la temperatura ambiente a la que se ve sometido el dispositivo. Por lo que, al no ser crítico el problema de la temperatura, se puede considerar como válida la simulación realizada.

\section{Conclusiones}

En este artículo verificamos la robustez en cuanto al ancho de línea del sensor o detector de longitud de onda propuesto por los autores. Los efectos en el ancho de línea en los láseres y sus causas son conocidos [17], y por eso aquí hemos demostrado que para la aplicación como sensor ese efecto no es un inconveniente. Tras esto, podemos seguir estudiando las posibles capacidades del sensor, puesto que no encontramos limitaciones a su aplicación.

\section{Agradecimientos}

A la Universidad Politécnica de Madrid por su apoyo al Grupo de investigación LST (Life Supporting Technologies). 